\title{
Sum Frequency Generation Confocal Microscopy Observation of a Fish Scale*
}

\author{
Wataru Kouyama, Atsushi Ogawa, Hongyan Li, Yoshihiro Miyauchi, and Goro Mizutani ${ }^{\dagger}$ \\ School of Materials Science, Japan Advanced Institute of Science \\ and Technology, Asahidai 1-1, Nomi, Ishikawa 923-1292, Japan \\ Haruyuki Sano \\ Ishikawa National College of Technology, Kitachujo, Tsubata, Ishikawa 929-0392, Japan
}

(Received 9 January 2014; Accepted 24 March 2014; Published 7 June 2014)

\begin{abstract}
An optical sum frequency generation (SFG) microscopy image of a fish scale of Pagrus major was observed. Its SFG spectra were also measured and were compared with that of collagen of Achilles tendon of a cow (Bos taurus). From this comparison, the peak near $2950 \mathrm{~cm}^{-1}$ in the fish scale spectrum was assigned to the fish collagen. The two collagen spectra showed different line shapes and widths owing to a difference in the background nonlinearity. In the SFG image of the fish scale cross section, stronger signal was observed from the sea side than from the body side.
\end{abstract}

[DOI: $10.1380 /$ ejssnt.2014.259]

Keywords: Sum frequency generation; Microscopy; Vibrational spectroscopy; Bioimaging and engineering; Biological molecules-proteins; Fish scale; Collagen

\section{INTRODUCTION}

It is known that infrared and Raman microscopes can visualize molecular vibration. On the other hand, sum frequency generation (SFG) microscopy uses the second order nonlinear effect. This effect is sensitive to the symmetry of material's structure because it occurs only at non-centrosymmetric parts in the sample. Hence this microscope is expected to be useful in the observation of biomaterials because most biomolecules have chiral or noncentrosymmetric structure. The spatial resolution of this microscope is determined by the diffraction limit at the observed wavelength. In order to realize a higher performance of the sum frequency generation microscopy, we developed a SFG microscopy incorporating a confocal optics [1]. However, this microscope has been developed only recently, and there are not many successful observation examples so far. Therefore, it is necessary to evaluate this microscopy's performance by observing as many samples as possible. For this purpose we used fish scales as a sample for evaluating the performance of the microscope. Fish scales contain collagen. Generally, a biomolecule is a chiral molecule, and thus collagen has second order nonlinearity. So far, optical second harmonic generation $[2,3]$, sum frequency generation spectrum [4], and SFG microscopic images [5] from collagen have been reported.

All multicellular organisms have collagen in themselves. Collagen is a protein based on glycine, proline, and hydroxyproline, and their chains are combined to form a triple-helical structure. Collagen is the main ingredient in the animal skin, bones, tendons, cartilage, and teeth. Because of such characteristics, the collagen is studied in the fields of anti-aging, food, cosmetics, healthy supplement, living body or medical materials such as an artificial cornea and vascular grafts [6]. General fish collagen has a low denaturation's temperature because it contains less

*This paper was presented at 9th International Symposium on Atomic Level Characterizations for New Materials and Devices, Sheraton Kona, Hawaii, U. S. A., December 2-6, 2013.

†Corresponding author: mizutani@jaist.ac.jp

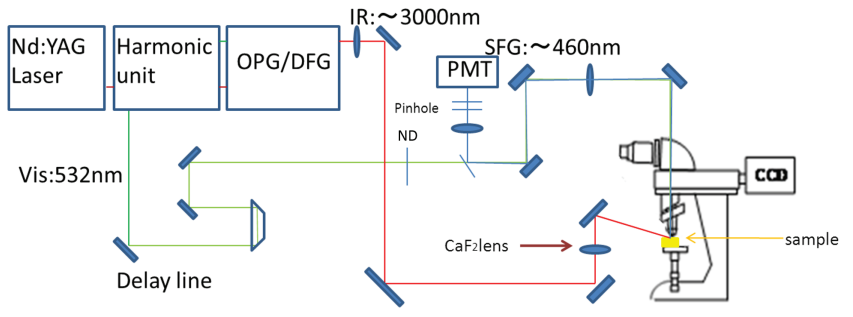

FIG. 1: Experimental Setting of the SFG confocal microscopy. OPG and DFG represent optical parametric generator and difference frequency generator, respectively. ND represents neutral density. PMT represents photomultiplier tube.

hydroxyproline contributing to structural stability $[7,8]$. However, fish collagen in scales is insoluble fibrillar collagen because collagen fibrillar skeletons are cross-linked together by minerals such as calcium. The fish scale has an analogous structure to human bones and it is studied with respect to the regenerative surgery of bones or teeth.

In this context, the fish scales are regarded as one of the best samples for the demonstration of our SFG confocal microscopy. In this study a scale of Pagrus major was used as the sample. There are two reasons for the choice of this species of fish. First, the scale of this fish is bigger than that of other fish and the cross section is easier to observe. Secondly, the Pagrus major is easily available in Japan so that we can take a good scale sample. We performed SFG microscopy and spectroscopy of the fish scale of Pagrus major.

\section{EXPERIMENTAL}

Figure 1 is the optical setting of our sum frequency generation confocal microscopy. The light source is a $\mathrm{Nd}^{3+}$ :YAG laser and the $1064 \mathrm{~nm}$ light from it is converted into a visible light of $532 \mathrm{~nm}$ wavelength in the harmonic unit. Therefore, infrared light of $3 \mu \mathrm{m}$ wavelength is generated in the optical parametric generator 


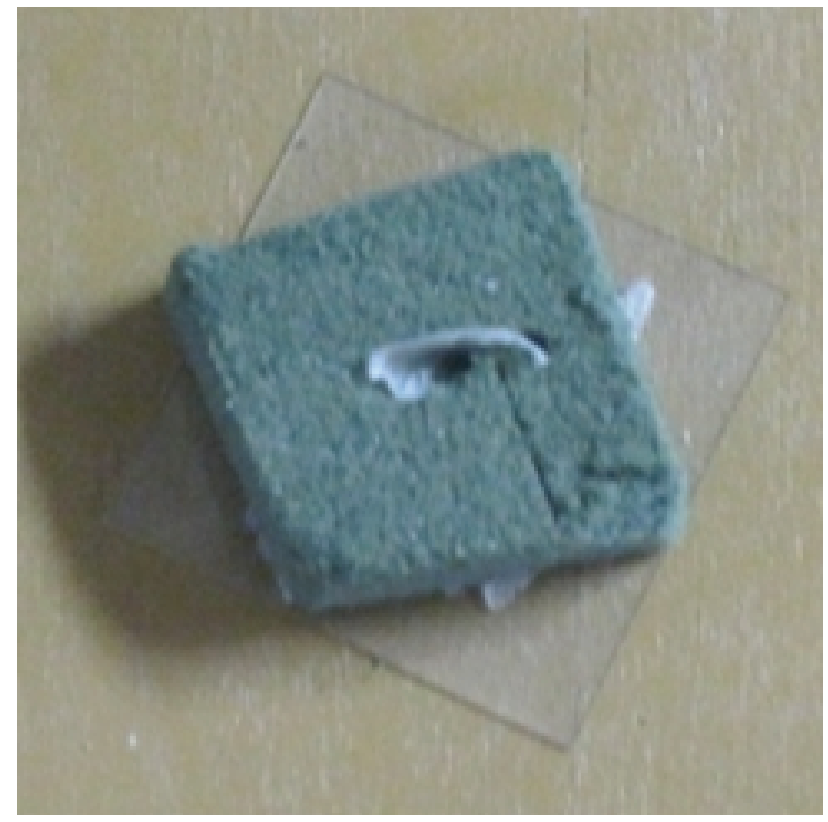

FIG. 2: Sample holder of the cross section of a fish scale.

and difference frequency generator (OPG/DFG) system using the $1064 \mathrm{~nm}$ and $532 \mathrm{~nm}$ light. This IR light was guided to the sample by using gold mirrors. The visible light of wavelength $532 \mathrm{~nm}$ passed the delay line and the neutral density (ND) filters, and irradiated the sample. The SFG light from the sample was collected by an objective lens $(\mathrm{NA}=0.45)$, reflected by a dichroic mirror, passed through band pass filters, was focused on a pinhole of $2 \mathrm{~mm}$ radius and detected by a photomultiplier.

The confocal optics has a spatial resolution $\sqrt{2}$ times better than a normal imaging optics by having a pinhole in its image-formation optics and blocking the off-focus light. The spatial resolution of $0.4 \mu \mathrm{m}$ was obtained for our SFG confocal microscope by using a $10 \mu \mathrm{m}$ pinhole [10]. However, since we could not obtain high SFG intensity from fish scale samples by using a $10 \mu \mathrm{m}$ pinhole, we used a $2 \mathrm{~mm}$ pinhole in this study. In this case the spatial resolution is determined by the focusing of the visible excitation beam by the objective lens and the lateral spatial resolution was around $2 \mu \mathrm{m}$. Correspondingly, the depth resolution was around $5 \mu \mathrm{m}$.

A water supply sponge (Daiso Sangyo Corp.) was used to hold the cross section of the fish scales as shown in Fig. 2. Bos taurus's (cow) Achilles tendon collagen was purchased from Wako Pure Chemical Industries, Ltd. and was used as delivered.

\section{RESULTS AND DISCUSSION}

Figures 3(a) and (b) are the SFG spectra of a fish scale cross section of Pagrus major at a position $20 \mu \mathrm{m}$ from the sea side edge and a position $30 \mu \mathrm{m}$ from the body side edge. The peaks are located around $2950 \mathrm{~cm}^{-1}$ and have rather symmetric shapes. Figure 3(c) shows an SFG spectrum of collagen from Bos taurus's (cow) Achilles tendon. The SFG peak of Bos taurus collagen is located also at

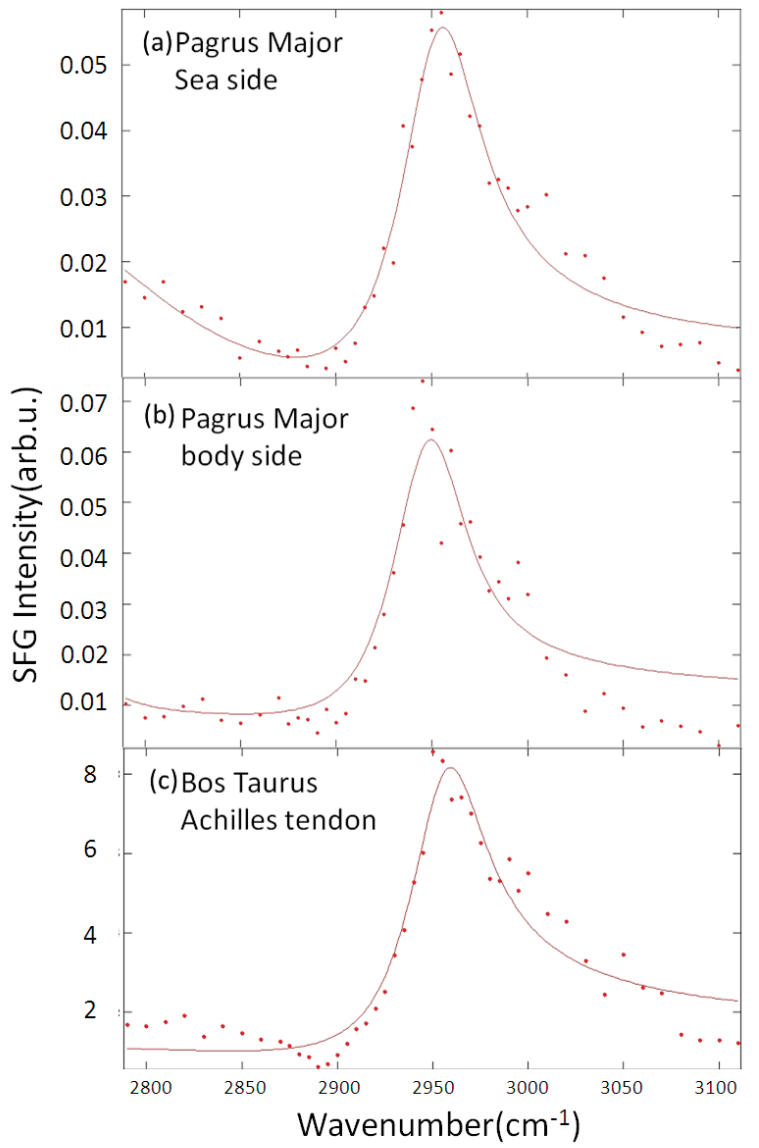

FIG. 3: SFG spectra of (a) Pagrus major fish scale crosssection near the sea side edge, and (b) near the body side edge, (c) Bos taurus Achilles tendon.

$2950 \mathrm{~cm}^{-1}$ but its shape has more asymmetric component than those in Figs. 3(a) and (b). This collagen is type I collagen [9]. The SFG spectrum of the collagen of Bos taurus's tendon in Fig. 3(c) is consistent with the previous report by Mendoza [4]. From the comparison among these three spectra, the SFG peaks in Figs. 3(a) and (b) are assigned to fish collagen.

The curves in Fig. 3 are the fitting curve according to the equation,

$$
\begin{aligned}
\left|\chi^{\mathrm{SFG}}\right|^{2}= & \left|\chi^{\mathrm{NR}}\right|^{2}+\frac{\left|\chi_{\mathrm{V}}\right|^{2}}{\left(\omega-\omega_{\mathrm{V}}\right)^{2}+\Gamma_{\mathrm{V}}^{2}} \\
& +2 \chi^{\mathrm{NR}} \frac{\chi_{\mathrm{V}}\left(\omega-\omega_{\mathrm{V}}\right)}{\left(\omega-\omega_{\mathrm{V}}\right)^{2}+\Gamma_{\mathrm{V}}^{2}} \cos \varphi \\
& -2 \chi^{\mathrm{NR}} \frac{\chi_{\mathrm{V}} \Gamma_{\mathrm{V}}}{\left(\omega-\omega_{\mathrm{V}}\right)^{2}+\Gamma_{\mathrm{V}}} \sin \varphi
\end{aligned}
$$

Here $\chi^{\mathrm{NR}}$ is a non-resonant background nonlinear susceptibility, $\omega_{\mathrm{V}}$ and $\Gamma_{\mathrm{V}}$ are the frequency and width of the resonant peak, respectively, $\varphi$ is the phase of the nonlinearity peak with respect to the background $\chi^{\mathrm{NR}}$, and $\chi_{\mathrm{V}}$ is the resonant nonlinear susceptibility. Table I shows the parameters used to draw the theoretical curves in Fig. 3. We can see in Table I that the peaks of the cow tendon and the fish scale spectra have similar positions and widths. 
TABLE I: Parameters used to reproduce the SFG intensity curves in Fig. 3.

\begin{tabular}{lccc}
\hline \hline & $\chi^{\mathrm{NR}} / \chi_{\mathrm{V}}\left(\times 10^{-3}\right)$ & Peak position & Peak width \\
\hline Pagrus major scale Sea side & $2.98 \pm 0.678$ & $2954 \pm 2.21 \mathrm{~cm}^{-1}$ & $85 \pm 29.5 \mathrm{~cm}^{-1}$ \\
Pagrus major scale body side & $2.54 \pm 0.227$ & $2948 \pm 0.00003 \mathrm{~cm}^{-1}$ & $81 \pm 0.00002 \mathrm{~cm}^{-1}$ \\
Bos Turus collagen & $3.54 \pm 0.702$ & $2956 \pm 2.7 \mathrm{~cm}^{-1}$ & $76 \pm 27.4 \mathrm{~cm}^{-1}$ \\
\hline \hline
\end{tabular}
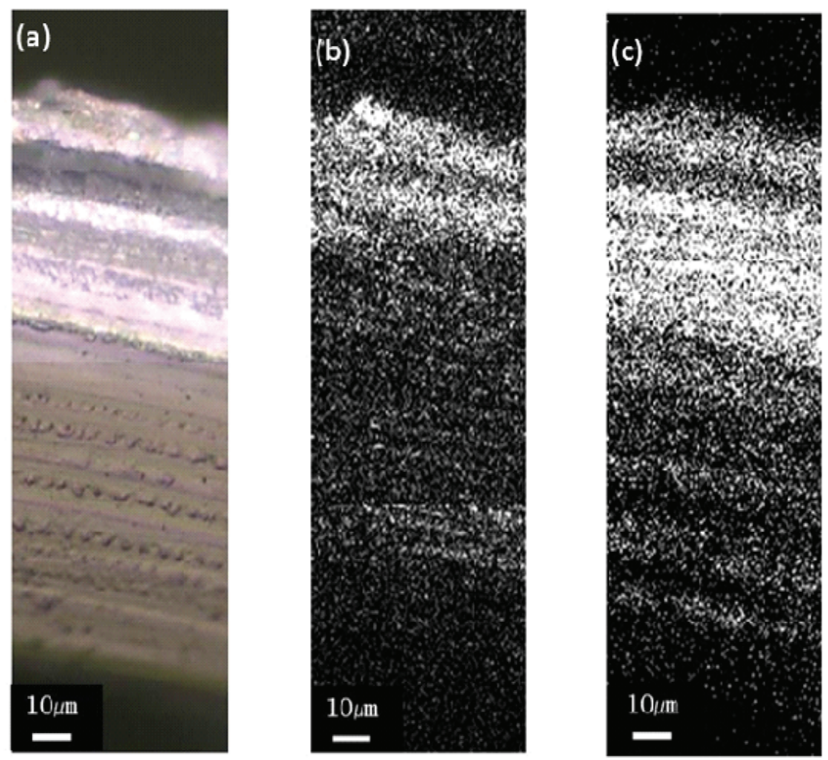

FIG. 4: Images of the cross section of a Pagrus major fish scale by using (a) a CCD camera (b) linear confocal microscope at the photon energy of $2.33 \mathrm{eV}$ (c) confocal SFG microscope at the infrared wave number of $2950 \mathrm{~cm}^{-1}$.

The peak position of the fish scale on the body side is a little lower in the frequency scale than those of the other two samples, but it is not significant because the shift is smaller than the peak widths. On the contrary, we can see in Table I that the non-resonant background $\chi^{\mathrm{NR}}$ of the nonlinear susceptibility of Bos taurus collagen was larger than that of fish the scale collagen. Especially, $\chi^{\mathrm{NR}} / \chi_{\mathrm{V}}$ of cow tendon collagen is larger than that of fish scale on the body side in a statistically meaningful way. Owing to this difference the SFG peak of the cow tendon looks broader and more asymmetric than that of the fish scale.

From the literature, the collagen of Bos taurus's tendon is different from that of the fish scale in the content of hydroxyproline and impurities [6]. Non-resonant components may originate from other materials such as lipid. These materials do not have their resonance in the present measurement range, and should contribute only to the background intensity of the spectra in Fig. 3.

Figure 4(a) shows a microscopic image of the cross section of the fish scale of Pagrus major taken by a CCD camera. Figure 4(b) is a linear confocal microscopic image of the same sample using $532 \mathrm{~nm}$ incident light. Figure 4 (c) is a corresponding SFG image by using the visible light of wavelength $532 \mathrm{~nm}$ and the IR light of $2950 \mathrm{~cm}^{-1}$ at the collagen's $\mathrm{CH}_{2}$ vibrational peak. White dots in the images in Fig. 4 are signal intensity points. When this fish scale sample was on the fish body, the sea water was in the upper direction of the figure (sea side), and the fish body was in the lower direction (body side). The image is bright in the upper part of both Fig. 4(b) and (c), but the dot distribution is different between the two images. In Fig. 4(c) the brightness distribution corresponds to that of collagen because infrared beam of $2950 \mathrm{~cm}^{-1}$ at collagen peak is used to obtain this SFG image. Since the sea side of the sample has more white dots than the body side, the sea side is interpreted to have more collagen ingredient.

Therefore, we are successful in observing fish collagen in scales by SFG confocal microscopy.

\section{CONCLUSIONS}

We have measured an SFG spectrum of collagen in a fish scale and observed its image by sum frequency generation microscopy. We compared SFG peak positions, peak widths and non-resonant components of cow collagen and the fish scale collagen. The difference was found in the non-resonant component. From the SFG intensity image we can say that the sea side part in the scale has more collagen content.

\section{Acknowledgments}

We thank Mr. Teraoka for providing us with the fish scale samples.
[1] K. Locharoenrat, H. Sano, and G. Mizutani, Phys. Status Solidi C 6, 304 (2009).

[2] S. Roth and I. Freund, J. Chem. Phys. 70, 1637 (1979).

[3] S. J. Lin, J. S. Chen, W. Lo, Y. Sun, W. L. Chen, J. Y. Chan, H. Y. Tan, W. C. Lin, C. J. Hsu, T. H. Young, S. H. Jee, and C. Y. Dong, Proc. SPIE 6084, 60840S (2006).

[4] R. Mendoza, D. R. Yankelevich, M. Wang, K. M. Reiser, C. W. Frank, and A. Knoesen, Biophys. J. 93, 4433 (2007).
[5] V. Raghunathan, Y. Han, O. Korth, N. H. Ge, and E. O. Potma, Optics Lett. 36, 3891 (2011).

[6] R. Duan, J. Zhang, X. Du, X. Yao, and K. Konno, Food Chem. 112, 702 (2009).

[7] Y. Nomura, H. Sakai, Y. Ishii, and K. Shirai, Biosci. Biotechnol. Biochem. 60, 2092 (1996).

[8] T. Ikoma, H. Kobayashi, J. Tanaka, D. Walsh, and S. Mann, Int. J. Biol. Macromolecules 32, 199 (2003).

[9] D. Voet, J. G. Voet, and C. W. Pratt, Fundamentals of 
Biochemistry, Third edition (Wiley, Hoboken, New Jersey, 2006), Chap. 6.

[10] N. A. Tuan, Y. Miyauchi, and G. Mizutani, Jpn. J. Appl.
Phys. 51, 122402 (2012). 